\title{
First Astronomical Use of Multiplexed Transition Edge Bolometers
}

\author{
D. J. Benford ${ }^{1}$, T.A. Ames ${ }^{1}$, J.A. Chervenak ${ }^{1}$, E.N. Grossman ${ }^{2}$, \\ K.D. Irwin ${ }^{2}$, S.A. Khan ${ }^{1,3}$, B. Maffei ${ }^{4}$, S.H. Moseley ${ }^{1}$, \\ F. Pajot ${ }^{5}$, T.G. Phillips ${ }^{6}$, J.-C. Renault ${ }^{7}$, C.D. Reintsema ${ }^{2}$, C. Rioux $^{5}$, \\ R.A. Shafer ${ }^{1}$, J.G. Staguhn ${ }^{1,8}$, C. Vastel $^{9}$ \& G.M. Voellmer ${ }^{1}$ \\ 1. NASA Goddard Space Flight Center, Greenbelt, MD 20771, USA \\ 2. NIST, Boulder, CO 80305 , USA \\ 4. University of Wales, Cardiff, CF24 3YB, Wales \\ 6. Caltech, Pasadena, CA 91125, USA \\ 8. Raytheon/ITSS, Lanham, MD 20706, USA \\ 3. Imperial College, London SW7 2BW, UK \\ 5. IAS-CNRS, 91405 Orsay, France \\ 7. IAP-CNRS, F-75014 Paris, France \\ 9. CESR, 31028 Toulouse, France

\begin{abstract}
We present performance results based on the first astronomical use of multiplexed superconducting bolometers. The Fabry-Perot Interferometer Bolometer Research Experiment (FIBRE) is a broadband submillimeter spectrometer that achieved first light in June 2001 at the Caltech Submillimeter Observatory (CSO). FIBRE's detectors are superconducting transition edge sensor (TES) bolometers read out by a SQUID multiplexer. The Fabry-Perot uses a low resolution grating to order sort the incoming light. A linear bolometer array consisting of 16 elements detects this dispersed light, capturing 5 orders simultaneously from one position on the sky. With tuning of the Fabry-Perot over one free spectral range, a spectrum covering $\Delta \lambda / \lambda=1 / 7$ at a resolution of $\delta \lambda / \lambda \approx 1 / 1200$ can be acquired. This spectral resolution is sufficient to resolve Doppler-broadened line emission from external galaxies. FIBRE operates in the $350 \mu \mathrm{m}$ and $450 \mu \mathrm{m}$ bands. These bands cover line emission from the important star formation tracers neutral carbon $[\mathrm{CI}]$ and carbon monoxide $(\mathrm{CO})$. We have verified that the multiplexed bolometers are photon noise limited even with the low power present in moderate resolution spectrometry.
\end{abstract}

\section{SCIENTIFIC MOTIVATION}

Spectroscopy of distant galaxies in the far-infrared and submillimeter has lagged behind continuum studies at the same wavelengths (e.g., with SHARC at the CSO [1]) and spectroscopic studies at longer wavelengths (e.g. OVRO [2]). In large part, this is due to a relative lack of available instrumentation combining high sensitivity and large bandwidth. Observing an emission line from a typical galaxy with velocity-broadened linewidth of $\sim 300 \mathrm{~km} / \mathrm{s}$ in the $350 \mu \mathrm{m}$ and $450 \mu \mathrm{m}$ atmospheric windows $(850 \mathrm{GHz}$ and $650 \mathrm{GHz}$, respectively) requires a spectrometer with a bandwidth of at least $0.5 \mu \mathrm{m}$ $(1 \mathrm{GHz})$. Additionally, detecting this line is easiest if the spectral resolution is approximately this width. Hence, a spectrometer with a spectral resolution of slightly

CP605, Low Temperature Detectors, edited by F. S. Porter et al. 2002 American Institute of Physics 0-7354-0049-0 589 
more than 1000 is optimal for the detection of faint galaxies in the far-infrared and submillimeter.

The Fabry-Perot Interferometer Bolometer Research Experiment, FIBRE, is an instrument designed to demonstrate a suite of advanced technologies suitable for sensitive detection of far-infrared light. This includes superconducting transition edge sensor (TES) bolometers, SQUID multiplexed amplifiers, and a cryogenic Fabry-Perot interferometer. These components are being developed for the SOFIA imaging FabryPerot spectrometer SAFIRE and for a complement of ground-based instruments.

\section{INSTRUMENT DESIGN}

\section{Superconducting TES Bolometers and SQUID Amplifiers}

The superconducting TES bolometer has been developed for use at wavelengths from the submillimeter to X-rays. It combines high speed with high sensitivity and can be read out by SQUID amplifiers, which are well suited to multiplexing. Unfortunately, the development of these detectors is too detailed to discuss at length here $[3,4]$. The FIBRE bolometers were used in the laboratory to demonstrate that multiplexed detection using the NIST-designed SQUID multiplexer of Chervenak et al. [4] was possible [5]. Further measurements, as presented by Staguhn et al. [6] in these proceedings, have verified that the noise performance of these detectors is limited by phonon and Johnson noise contributions as predicted by theory.

FIBRE features two $1 \times 8$ monolithic bolometer arrays consisting of $1 \mathrm{~mm} \times 1 \mathrm{~mm}$ absorbers with a $50 \mu \mathrm{m} \times 150 \mu \mathrm{m} \mathrm{Mo/Cu}$ bilayer TES. This is shown in Fig. 1 .

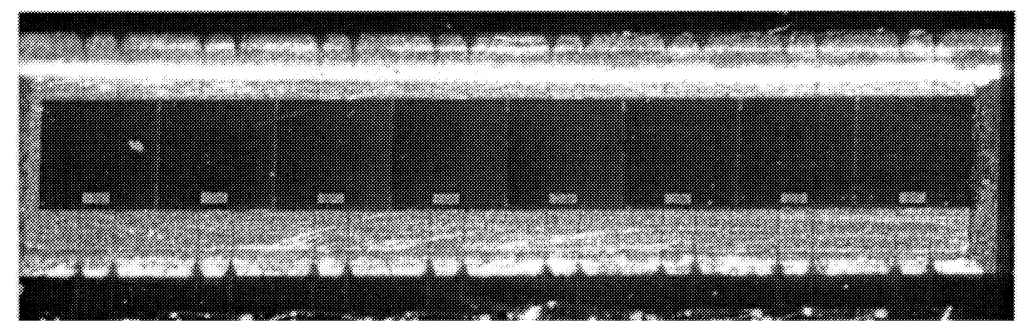

FIGURE 1. Photograph of a single $1 \times 8$ monolithic bolometer array. Each $1 \mathrm{~mm} \times 1 \mathrm{~mm}$ pixel is a $1 \mu \mathrm{m}$ thick silicon membrane supported by 4 legs approximately $5 \mu \mathrm{m}$ wide. The TES is the small pale rectangle at the bottom center of each detector.

\section{Optical Design}

The optical design uses a single Fabry-Perot etalon followed by an order-sorting grating [7]. The grating is blazed to operate in its first order, which is broad enough to transmit orders $40-45$ of the Fabry-Perot for $350 \mu \mathrm{m}$ operation and orders 32-35 for $450 \mu \mathrm{m}$ operation. The grating disperses these Fabry-Perot orders along the array such that they are separated onto adjacent sets of pixels. In this manner, a spectrum consisting of several orders of the Fabry-Perot is collected simultaneously. By 
stepping the Fabry-Perot over one free spectral range, a complete spectrum is accumulated.
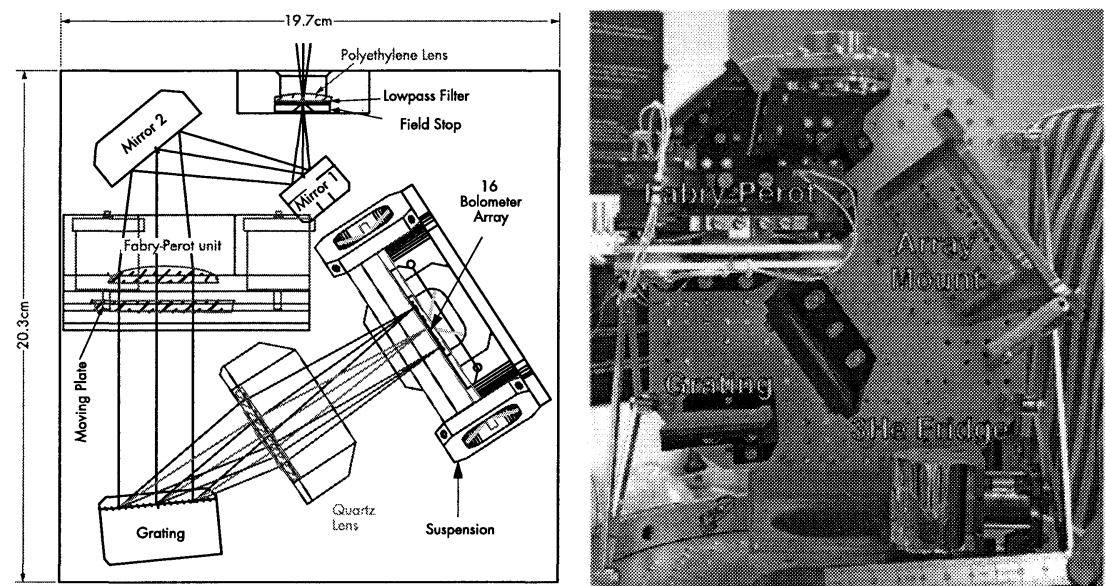

FIGURE 2. (Left) Diagram of spectrometer optics, showing three orders being dispersed onto the bolometer array. (Right) Optics being assembled, with the detector array and baffles yet to be added.

\section{Commissioning Observations}

FIBRE was delivered to the Caltech Submillimeter Observatory (CSO) on Mauna Kea, Hawai'i in May 2001. During six nights of poor weather, the instrument was operated and found to work very well. The bolometers were read out in multiplexed fashion (Fig. 3). Those bolometers that were illuminated at a given Fabry-Perot tuning were found to have $\sim 20$ times the noise of the dark bolometers. The expected photon noise contribution is approximately 10 times the intrinsic (phonon + Johnson) noise of the detectors, so the system noise is near the theoretical performance and the bolometers are background-limited with a net NEP of $3 \times 10^{-17} \mathrm{~W} / \sqrt{ } \mathrm{Hz}$. A spectrum (Fig. 4) was taken using a local oscillator source operating at $372 \mu \mathrm{m}(807 \mathrm{GHz})$. The spectral resolving power was measured to be 1200 , for a velocity resolution of $250 \mathrm{~km} / \mathrm{s}$, as predicted from the known performance of the Fabry-Perot.

The opacities at the zenith during the observing run were measured by skydips using both FIBRE and the CSO facility $350 \mu \mathrm{m}$ taumeter. These measurements yielded zenith opacities of $\tau_{350 \mu \mathrm{m}} \sim 4$ during most of the run. No scientific data could be taken in such poor conditions, but in order to demonstrate a multiplexed detection using TES bolometers, we

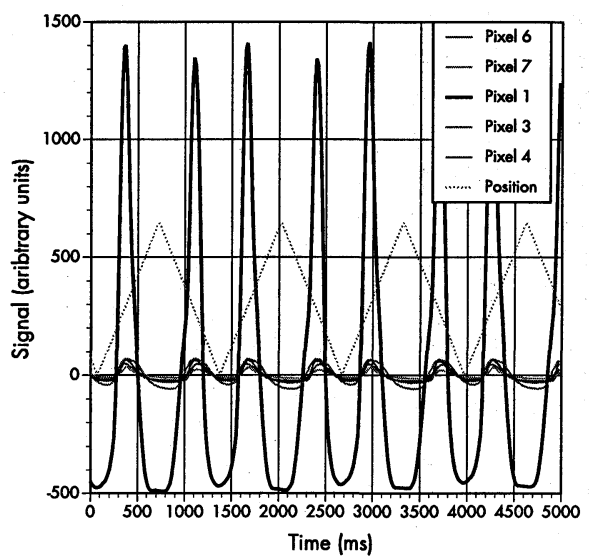

FIGURE 3. Multiplexed readout of the FIBRE bolometers while tuning the Fabry-Perot and observing a local oscillator source. The signal is seen strongly on one channel, weakly in others, demonstrating good optical performance for 
observed the limb of the Moon at $365 \mu \mathrm{m}$.

spectroscopy.

The secondary mirror was nutated to subtract the atmosphere, so we obtained a high signal-to-noise detection of the Moon emission despite a transmission of $\sim 1 \%$ (Fig. 5).

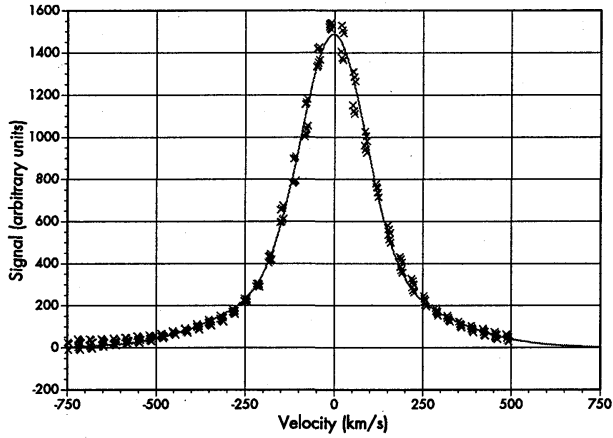

FIGURE 4. Calibration spectrum taken using a local oscillator operating at $372 \mu \mathrm{m}(807 \mathrm{GHz})$, calibrated in velocity units. The resolution is $250 \mathrm{~km} / \mathrm{s}$, for a resolving power of 1200 .

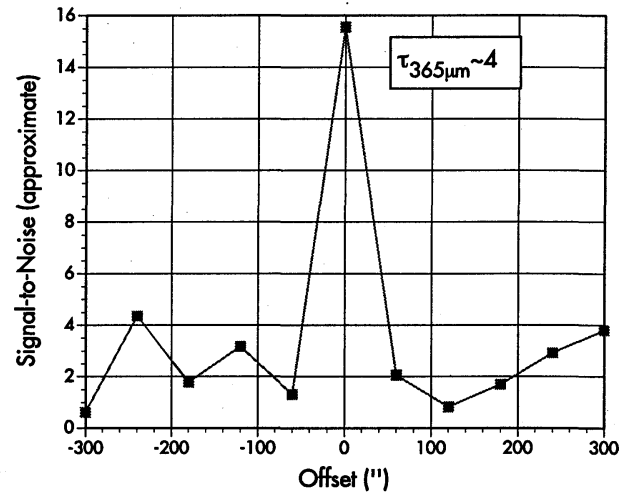

FIGURE 5. Detection of the limb of the Moon, taken while chopping so that signal is seen only when exactly on the limb. Each data point contains 3 seconds of on-source time.

\section{CONCLUSION}

FIBRE achieved first light at the CSO, detecting the Moon at $365 \mu \mathrm{m}$ in bad weather with an atmospheric transmission of $\sim 1 \%$. The spectrometer was operating with a spectral resolving power of $\sim 1200$, and the signal amplitude and noise were consistent with expectations. The TES bolometer and SQUID multiplexer technology has been thus validated in an astronomical application. We anticipate future observations to study galaxies in the fine-structure line of $\mathrm{CI}$ and the $\mathrm{CO}$ rotational lines, and to continue to refine the multiplexed TES detectors in astronomical applications.

\section{ACKNOWLEDGMENTS}

We thank the staff of the CSO for making the observations described here possible; their support was crucial in the commissioning of FIBRE. We owe a debt of gratitude to many at NASA/GSFC for their contributions to FIBRE hardware and software.

\section{REFERENCES}

1. Benford, D.J., Cox, P., Omont, A., Phillips, T.G. \& McMahon, R.G., ApJ L518, 65 (1999).

2. Blain, A.W., Frayer, D.T., Bock, J.J., \& Scoville, N.Z., MNRAS 313 (3), 559-570 (2000).

3. Benford, D.J. et al., ASP Conference Series 217, 134-139 (1999).

4. Chervenak, J.A, et al, Appl. Phys. Letters 74 (26), 4043 (1999).

5. Benford, D.J., et al., Int. J. IR MM Waves 21 (12), 1909-1916 (2000).

6. Staguhn, J.G., et al., these proceedings.

7. Maffei, B., et al., Infrared Physics \& Technology 35, 321 (1994). 